\title{
Resource Scheduling Simulation Design of Firefly Algorithm Based on Chaos Optimization in Cloud Computing
}

\author{
Yue Miao \\ (China West Normal University, School of Computer, Sichuan Nanchong 637002)
}

\begin{abstract}
The problem of resources under the environment of cloud computing has always been the focus of research. In this paper, the Artificial Firefly Algorithm is studied, on the basis of which the chaos algorithm is introduced to improve the algorithm with regard to the problem of the subsequent search and optimization precision deficiency cause by the lack of initialization of firefly position in the firefly algorithm. Meanwhile, the updating of luciferin is improved. The improved algorithm is enhanced greatly in accuracy and performance. Through the simulation platform Cloudsim, it is found that under the cloud computing model, the algorithm presented in this paper can effectively reduce the average time spent by subtasks in processing request tasks, and thus improve the efficiency of task processing and achieve a rational allocation of resources.
\end{abstract}

Keywords: Artificial Firefly Algorithm, cloud computing, resource allocation, chaos algorithm

\section{Introduction}

Cloud computing is developed from the three major computing methods of grid computing, distributed computing and parallel computing [1]. Users only need to search and query the resources they need via the PC in cloud computing. In the process of search and query, various resources in cloud computing are required. How to make computing resources allocation is a very important issue. Literature [2] proposed a multi-objective comprehensive evaluation model of virtual machine resource scheduling, and applied the typical multi-objective optimization algorithm in the problem, to combine the virtual machine resources scheduling and task allocation into a single process, to reduce the complexity of the problem. Simulation comparison experiment results show that the method can provide multiple candidate solutions by solving once, with good usability and scalability. The literature [3] proposed the building of cloud computing platform based on the Cloudsim open source framework, as well as three resource allocation strategies based on fault-tolerant QoS, random allocation and sequential distribution, and compared the three strategies presented. The experimental results show that the virtual machine resources allocation strategy based on fault-tolerant QoS has advantages in performance. Literature [4] analyzed the resources scheduling of virtual machine, to classify the cloud service resources pool into saturated pool and unsaturated pool; and then provide resource scheduling strategies respectively on the state of cloud service resources pool under the current task request, and verify the validity of the method presented through analysis of computing examples. The virtual machine resource scheduling method presented will contribute to a balanced use of virtual machine resources in the cloud services resource pool. Literature [5] proposed a resource allocation mechanism of self-management, self-adjustment and self-protection in cloud computing resources, which 
has certain advantages. Literature [6] proposed a resource allocation system for large-scale distributed systems, which effectively improves the quality of the system service in cloud computing. Literature [7] proposed the combination of attraction exclusion mechanism in the internal searching strategies of adding subgroups in the leapfrog algorithm in cloud computing, to avoid premature convergence of the algorithm. In this paper, on the basis of research on Artificial Firefly Algorithm, with regard to the lack of initialization of artificial fireflies' positions, the chaos algorithm is introduced, and the updating of luciferin is improved. The improved algorithm can better adapt to the issue of resource allocation in cloud computing. The simulation results show that the improvement of the algorithm is effect to a certain extent, improving the efficiency of the algorithm processing tasks and reducing the time consumed by the network.

\section{Resource Status under Cloud Computing}

In the cloud environment, it is required to timely process the number of resources used by each cloud user, time occupied and network access charges. Currently, the task scheduling algorithm involved mainly focuses on whether the total time required to complete the task is minimized, while few has considered the time cost for finishing the subtasks. This results in the misallocation of time and resources in the multiple tasks performed by the cloud users. In this paper, in order to better describe the status of cloud computing, make the following assumptions:

(1) The larger tasks are separated into a certain number of subtasks, to make the execution time of each subtask of little difference as far as possible.

(2) The quantity of resources allocated meets the demands of subtasks.

(3) Reasonably define the time during which the sub-tasks occupy the resources so that the time spent on each subtask is of little difference.

At the same time, as cloud customers, the following constraints shall be made:

(1) Set a certain sequence for the requests of cloud customers

(2) Each customer task can only be responded simultaneously by several cloud servers, and once started, it can not be interrupted.

(3) The priority of each task is not taken into account.

\section{Artificial Firefly Algorithm}

Artificial Firefly Algorithm is a resource scheduling algorithm to face the parallel conditions. The objective functions required to be solved is modeled as individual fireflies, to find the optimal arrangement of resources by solving the optimal individual firefly. In this algorithm, each firefly is distributed in the defined space of the objective function, and each has its own visual range, the area of which will be influenced by the adjacent fireflies. When there are a small number of fireflies around, the visual range of a firefly will increase, so it will attract more fireflies. On the contrary, its visual range will become smaller. The place where there are the most fireflies will be the optimal solution of algorithm.

Assuming the firefly cluster is $N$, the position $\left(x_{i}, y_{i}\right)$ of the number $i$ firefly corresponds to the objective function $f\left(\left(x_{i}, y_{i}\right)\right)$ and luciferin value of the firefly is $T_{i}$, and the updating formula of each firefly's visual range is: 


$$
R_{d}^{i}(t+1)=\min \left\{R_{s}, \max \left\{0, R_{d}^{i}(t)+\beta\left(n_{t}-\left|N_{i}(t)\right|\right)\right\}\right\}
$$

In the formula, $R_{d}^{i}(t+1)$ is the visual range of the number $i$ firefly at $t+1, R_{s}$ is the range of perception, and $n_{t}$ is the threshold of the number of firefly neighbors. $\beta$ is the control constant, and $N_{i}(t)$ is the number of fireflies with high fluorescent within the visual range. Among them, the formula of $N_{i}(t)$ is as follows:

$$
N_{i}(t)=\left\{j:\left\|x_{j}(t)-x_{i}(t)\right\|<R_{d}^{i} ; l_{i}(t)<l_{j}(t)\right\}
$$

Wherein, $x_{j}(t)$ represents the position of the number $j$ firefly in the number $t$ generation, $l_{j}(t)$ represents the value of luciferin of the number $j$ firefly in the number $t$ generation, and the position distance between adjacent fireflies is within the visual range $R_{d}^{i}$.

The selection probability of firefly neighbors is:

$$
p_{i j}(t)=\frac{l_{j}(t)-l_{i}(t)}{\sum_{k \in N_{i}(t)} l_{k}(t)-l_{i}(t)}
$$

The updating formula of the position of fireflies:

$$
x_{i}(t)=x_{i}(t-1)+s\left(\frac{x_{j}(t-1)-x_{i}(t-1)}{\left\|x_{j}(t-1)-x_{i}(t-1)\right\|}\right)
$$

Luciferin value formula:

$$
l_{i}(t+1)=(1-\rho) l_{i}(t)+\gamma J\left(x_{i}(t+1)\right)
$$

In equation (5), $\gamma$ is the parameter for measuring the function value, and $J\left(x_{i}(t+1)\right)$ is the fitness value of functions.

In nature, it is assumed that firefly $i$ enters the visual range of firefly $j$, whose luciferin value is greater than that of firefly $i$. According to the probability $p_{i j}(t)$, firefly $i$ selects firefly $j$, and after the selection is completed, the location and luciferin of firefly $i$ is updated, and then calculate the objective function value of the current position.

\section{The Algorithm Presented in this Paper Studied in Cloud Computing Tasks}

\subsection{The Idea of the Algorithm of this Paper Improved}

In Artificial Firefly Algorithm, as the location of individual firefly is not defined in the process of algorithm initialization, it results in a waste of time in the movement of individual firefly to a certain extent. In this paper, the initialization of individual fireflies is first set by introducing the chaos algorithm, so that individuals initialized can be distributed reasonably. Moreover, the value of luciferin is an important reference factor for determining the current location of fireflies. This is because when the firefly with a high value of luciferin can guide 
other fireflies get close to it, and thus the target value of the function can be quickly identified. However, the current firefly algorithm is easy to fall into local optimum. In respect to these problems, this paper improves the initialization position and fitness value of luciferin. First of all, chaotic initialization is carried out on the location of fireflies, and then improvement is made with regard to equation (5).

1. The improvement of the location initialized

The chaos algorithm has always been an effective method for solving the nonlinear problems. In this paper, the chaos algorithm is introduced in the location initialization, which enables the artificial fireflies to find better progress directions after initialization, and the Tent mapping of the chaotic variable is:

$$
x_{i+1}= \begin{cases}2 x_{i} & x_{i} \in\left[\begin{array}{ll}
0 . & 0.5
\end{array}\right] \\
2\left(1-x_{i}\right) & x_{i} \in\left[\begin{array}{ll}
0.5 & 1
\end{array}\right]\end{cases}
$$

In the Artificial Firefly Algorithm, the state of the number $i$ firefly in the $k$-dimensional space is represented as $X_{i k}$, and mapped to the section $[0,1]$ according to equation (6)

$$
f x_{i k}=\frac{\left(x_{i k}\right)}{\left(\max _{k}+\min _{k}\right) / 2}
$$

In the equation, $\min _{k}$ and $\max _{k}$ represent the minimum and maximum values of variable $x_{i k}$ in the $k$-dimensional space, respectively.

After $m$ times of iterations of formula (7), the state value in the chaotic state is mapped according to (8), to get the new chaotic value (9).

$$
\begin{aligned}
& f x_{(i+1) k}^{s}=f x_{i k}^{s}\left(\max _{k}+\min _{k}\right) / 2 \\
& f x_{i}^{s}=\left(f x_{i 1}^{s}, f x_{i 2}^{s}, \ldots, f x_{i m}^{s}\right)
\end{aligned}
$$

Equation (9) is a description of the sequence after the chaotic sequence is carried out for individual artificial fireflies. Such a sequence is helpful to the subsequent movement of fireflies, and can reduce the unnecessary moving time.

2. The improvement of the fitness value of fireflies

The fitness value of fireflies is obtained from formula (5), and a parameter $\lambda$ is introduced in this paper to improve the value, to get formula (10)

$$
\left\{\begin{array}{l}
l_{i}(t)=l_{i}(t-1)+d \gamma J\left(x_{i}(t)\right) \\
d=1-e^{-\lambda \pi r_{s}^{2}}
\end{array}\right.
$$

In formula (10), $r$ represents the minimum radius set between fireflies, and $\lambda$ means the density of fireflies.

The two aspects of improvement mentioned above allow the improved Artificial Firefly Algorithm to effectively prevent the algorithm from falling into local convergence, and to quickly find the best position, so it is more in line with the rational use of multi-task resources in cloud computing. From formula (6) and (7), it is found that after using chaos algorithm to initialize the location of fireflies, it can guarantee the more reasonable completion time 
between tasks of the cloud computing server, and effectively reduce the time wasted. By the improvement of the fitness value in formula (10), the too fast convergence rate is controlled to some extent in the improved firefly algorithm. Therefore, the value of objective function can be solved to a minimum.

\subsection{Procedures of the Algorithm}

(1) Initialize the various parameters in the algorithm, introduce the chaos algorithm for the position of fireflies, and define the minimum radius and density values of fireflies.

(2) Calculate the new value of luciferin of each firefly according to formula (10), and the objective function of the fireflies is the value of luciferin.

(3) Calculate the new value of luciferin according to formula (10), and control it by using formula $1-e^{-\lambda \pi r_{s}^{2}}$.

(4) Change the positions of the fireflies, and select the eligible fireflies according to formula (2).

(5) Select a firefly $i$ in the moving direction randomly, and update it according to formula (4).

(6) After a completion of iteration and in the process of entering the next iteration, it is determined whether the number of iterations meets the termination condition. If it does not, go to step 2 . If it does, directly output the optimal solution.

\section{Experimental Simulation}

The CloudSim [8] platform, Core i3CPU and 2GDDR3, the operating system of Windows $\mathrm{xp}$ are adopted and matlab2012 is used in simulation, so as to better validate the effect of the algorithm presented in this paper in the resources allocation of cloud computing. The number of fireflies is set as 200, the number of tasks in cloud computing is 900, predicted transmission time is $100 \mathrm{~ms}$, the execution time is $300 \mathrm{~ms}$, and the number of iterations is 200 . The algorithm of this paper and other algorithms are compared, and it is found the algorithm of this paper performs better in the consumption of network and time.

1. The algorithm of this paper and Artificial Firefly Algorithm.

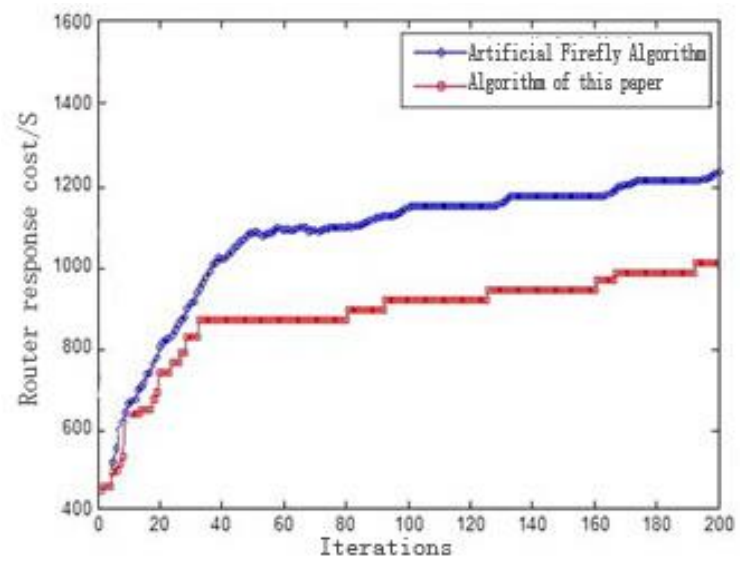

Figure 1. Comparison of the Routing Response of the Two Algorithms 
From Figure 1, it can be seen that the algorithm presented in this paper tends to be steady when the number of iterations gradually increases, and in terms of the routing network consumption, this algorithm is better than the basic Artificial Firefly Algorithm by $18 \%$. This means the algorithm presented in this paper has certain advantages.

2. Comparison of this algorithm and algorithms in other literature.

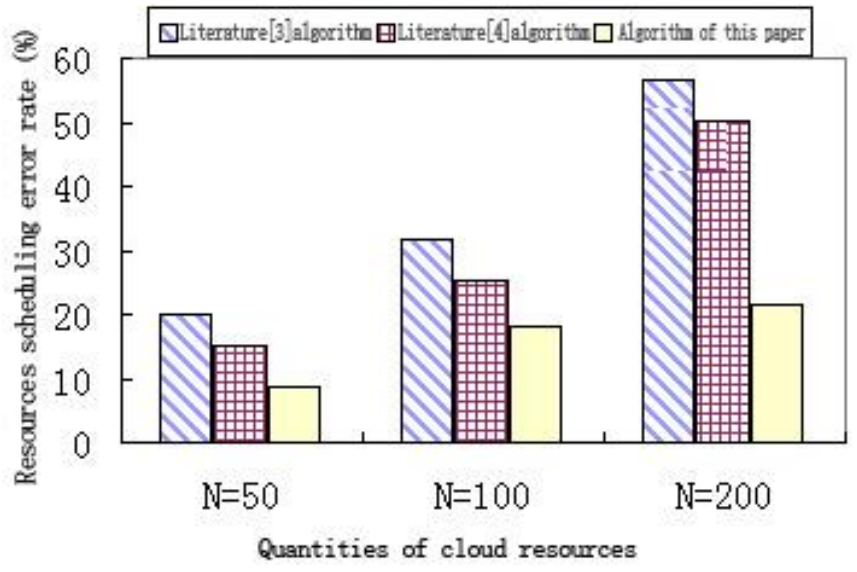

Figure 2. Comparison of Three Algorithms in Terms of Resource Scheduling Time

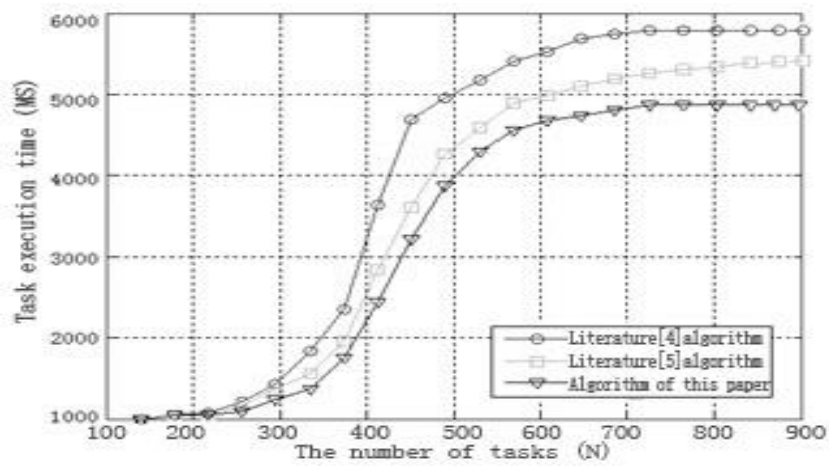

Figure 3. Comparison of Three Algorithms in Terms of Resource Execution Time

From Figure 2-3, it can be found that along with the gradual increase in the number of sub-tasks, the algorithm of this paper shows certain advantages in the aspects of task scheduling error rate and execution time. It is mainly because the improved Artificial Firefly Algorithm can better allocate resources in the cloud computing model. The execution time of the algorithm presented in this paper is close to the actual completion time, which meets the task allocation time in the cloud computing environment.

\section{Conclusion}

It has always been a major orientation of research how to better carry out the resource scheduling in the cloud computing environment. In this paper, the Artificial Firefly Algorithm and resources scheduling in cloud computing are combined together. With regard to the fast 
convergence rate and inaccuracy in solving, first the chaos algorithm is introduced to improve the initial position of fireflies, which effectively reduces the time consumption of the improved algorithm. Moreover, with the strategy of improving the position of firefly luciferin, the fireflies are enabled to quickly find good targets. However, it must be admitted that there are many realistic problems to solve in cloud computing, and the problem of resources allocation in cloud computing is worth further studying.

\title{
References
}

[1] I. Foster, Y. Zhao and I. Raicu, "Cloud computing and grid computing 360-degree compared", Proceeding of the 2008 Grid Computing Environments Workshop, Washington, DC: IEEE Computer Society, (2008), pp. $1-10$.

[2] X. Bo, Z. Chao and Z. Yanjun, "Multi-target optimization of virtual machine resources scheduling in cloud computing”, Journal of System Simulation, vol. 26, no. 3, (2014), pp. 592-595.

[3] B. Fei and W. Yun, "Virtual machine resources allocation strategy based on fault-tolerant QoS in cloud computing", Microelectronics and Computer, vol. 30, no. 3, (2013), pp. 136-139.

[4] L. Jihan, L. Sufen and Z. Yunyong, "Research on virtual machine resources balanced allocation methods in cloud computing environment", Telecommunications Science, vol. 29, no. 4, (2013), pp. 78-82.

[5] M. Randles, A. Taleb-Bendlab and D. Lamb, "Scalable self-governance using service commuities as ambients", Proceedings of SERVICES'09 Proceedings of the 2009 Congress on Services-I. Washington, DC: IEEE Computer Society, (2009), pp. 813-820.

[6] O. Abu-Rahmeh, P. Johnson and A. Taleb-Bendiab, "A dynamic biased random sampling scheme for scalable and reliable grid networks", Journal of Computer Science, vol. 7, no. 4, (2008), pp. 1-10.

[7] Z. Pengjun, "SFLA for solving the complex functions optimization problem", Application Research of Computers, vol. 26, no. 7, (2009), pp. 2435-24 37.

[8] R. N. Calheiros, R. Ranjan, R. Cafd and R. Buyya, CloudSim: a novel framework for modeling and simulation of cloud computing infrastructures and services [EB/OL]. [2009-9-3].

\begin{abstract}
Author
Yue Miao (1980 ), Master, Lecturer, engaged in the research on computer network and computer simulation.
\end{abstract}


International Journal of Grid Distribution Computing Vol.7, No.6 (2014) 